\title{
Differentially regulated miRNAs as prognostic biomarkers in the blood of primary CNS lymphoma patients
}

\author{
Roth, P ; Keller, A ; Hoheisel, J D ; Codo, P ; Bauer, A S ; Backes, C ; Leidinger, P ; Meese, E ; Thiel,
} E ; Korfel, A ; Weller, M

\begin{abstract}
Despite improved therapeutic regimens, primary CNS lymphoma (PCNSL) remains a therapeutic challenge. A prognostic classification of PCNSL patients may represent an important step towards optimised patient-adapted therapy. However, only higher age and low Karnofsky Performance Status (KPS) have repeatedly been reported to be associated with shorter overall survival (OS). Here we characterised microRNA (miRNA) fingerprints in the blood of PCNSL patients with short-term survival (STS) versus long-term survival (LTS) to assess their potential as novel prognostic biomarkers. Blood was collected from patients enrolled in the G-PCNSL-SG1 trial, a phase III study for patients with newly diagnosed PCNSL. miRNAs were extracted from the blood and analysed by next generation sequencing. The STS group comprised 20 patients with a median OS of 3months and was compared to 20 LTS patients with a median OS of 55 months. The cohorts were balanced for age and KPS. Twelve annotated miRNAs were significantly deregulated between the two groups. Among them, miR-151a-5p and miR-151b exhibited the most prominent differences. Importantly, the combination of several miRNA allowed for a good separation between short- and long-term survivors with maximal Area Under Curve (AUC) above 0.75. Besides the known miRNAs we identified putative novel miRNA candidates with potential regulatory influence of PCNSL. Finally, the differential regulation of the most promising candidate miRNAs was confirmed by real-time polymerase chain reaction (PCR) in a validation cohort consisting of 20 STS and LTS patients. In conclusion, peripheral blood miRNA expression patterns hold promise as a prognostic tool in PCNSL patients.
\end{abstract}

DOI: https://doi.org/10.1016/j.ejca.2014.10.028

Posted at the Zurich Open Repository and Archive, University of Zurich

ZORA URL: https://doi.org/10.5167/uzh-104737

Journal Article

Accepted Version

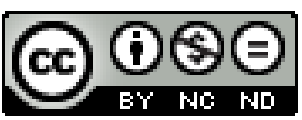

The following work is licensed under a Creative Commons: Attribution-NonCommercial-NoDerivatives 4.0 International (CC BY-NC-ND 4.0) License.

Originally published at:

Roth, P; Keller, A; Hoheisel, J D; Codo, P; Bauer, A S; Backes, C; Leidinger, P; Meese, E; Thiel, E; Korfel, A; Weller, M (2015). Differentially regulated miRNAs as prognostic biomarkers in the blood of primary CNS lymphoma patients. European Journal of Cancer, 51(3):382-390. 
DOI: https://doi.org/10.1016/j.ejca.2014.10.028 
Differentially regulated miRNAs as prognostic biomarkers in the blood of PCNSL patients

Patrick Roth $^{\mathrm{a} * \ddagger}$, Andreas Keller $^{\mathrm{b}}$, Jörg D. Hoheisel ${ }^{\mathrm{c}}$, Paula Codo $^{\mathrm{a}}$, Andrea S. Bauer $^{\mathrm{c}}$, Christina Backes $^{\mathrm{d}}$, Petra Leidinger ${ }^{\mathrm{d}}$, Eckart Meese $^{\mathrm{d}}$, Eckhard Thiel $^{\mathrm{e}}$, Agnieszka Korfel $^{\mathrm{e}}$, Michael Weller ${ }^{\mathrm{a}}$

${ }^{\mathrm{a}}$ Department of Neurology, University Hospital Zurich, 8091 Zurich, Switzerland, ${ }^{\mathrm{b}}$ Chair for Clinical Bioinformatics, Saarland University, 66123 Saarbrücken, Germany, ${ }^{\mathrm{c}}$ Division of Functional Genome Analysis, German Cancer Research Center, 69121 Heidelberg, Germany, ${ }^{\mathrm{d}}$ Department of Human Genetics, Saarland University, 66421 Homburg, Germany, ${ }^{\mathrm{e}}$ Department of Hematology and Oncology, Charité, 12200 Berlin, Germany

These two authors contributed equally

*Correspondence: Dr. Patrick Roth, Department of Neurology, University Hospital Zurich, Frauenklinikstrasse 26, 8091 Zurich, Switzerland, Tel.: +41 (0)44 255 5500, Fax: +41 (0)44 255 4507, E-mail: patrick.roth@usz.ch

Running title: Blood-derived miRNA profiles in PCNSL

Funding: This work was supported by a grant from the Hartmann Müller Foundation to PR. 


\section{ABSTRACT}

Despite improved therapeutic regimens, primary CNS lymphoma (PCNSL) remains a therapeutic challenge. A prognostic classification of PCNSL patients may represent an important step towards optimized patient-adapted therapy. However, only higher age and low Karnofsky Performance Status (KPS) have repeatedly been reported to be associated with shorter overall survival (OS).

Here we characterized microRNA (miRNA) fingerprints in the blood of PCNSL patients with short-term survival (STS) versus long-term survival (LTS) to assess their potential as novel prognostic biomarkers. Blood was collected from patients enrolled in the G-PCNSL-SG1 trial, a phase III study for patients with newly diagnosed PCNSL. miRNAs were extracted from the blood and analyzed by next generation sequencing.

The STS group comprised 20 patients with a median OS of 3 months and was compared to 20 LTS patients with a median OS of 55 months. The cohorts were balanced for age and KPS. Twelve annotated miRNAs were significantly deregulated between the two groups. Among them, miR-151a-5p and miR-151b exhibited the most prominent differences. Importantly, the combination of several miRNA allowed for a good separation between short- and long-term survivors with maximal AUC above 0.75. Besides the known miRNAs we identified putative novel miRNA candidates with potential regulatory influence of PCNSL. Finally, the differential regulation of the most promising candidate miRNAs was confirmed by real-time PCR in a validation cohort consisting of 20 STS and LTS patients.

In conclusion, peripheral blood miRNA expression patterns hold promise as a prognostic tool in PCNSL patients.

Key words: Primary CNS lymphoma, microRNA, biomarker, prognostic factor, next generation sequencing 


\section{INTRODUCTION}

Primary CNS lymphoma (PCNSL) is a malignant tumor, which is typically characterized by rapid growth causing severe clinical symptoms. In contrast to many other primary or secondary malignant brain tumors, PCNSL is a curable disease, at least in young patients who can tolerate aggressive therapeutic approaches. High-dose methotrexate (HD-MTX) is regarded the backbone of all current treatment strategies. The addition of various other drugs such as cytarabine, ifosfamide and high-dose chemotherapy with subsequent stem cell support has been assessed or is under clinical investigation $(1,2)$. Higher age and reduced performance status have repeatedly been described as prognostic factors associated with shorter survival (3-5). The definition of additional prognostic factors might be helpful to define patient populations that require an even more intense or prolonged therapy to delay tumor recurrence and unfavourable outcome. However, the search for other parameters supporting a prognostic discrimination has been widely futile except for some tissue markers including the expression of bcl-6 which has been associated with a favourable course of the disease (6). Furthermore, it has been reported that intraocular involvement in PCNSL may represent a negative prognostic indicator (7). In contrast, the relevance and application of minimally invasive biomarkers such as blood-borne patterns (e.g. LDH) has been discussed controversially (4).

microRNAs (miRNA) are short non-coding RNA molecules which act as post-transcriptional

regulators of gene expression (8) and are frequently deregulated under pathological conditions (9). Little is known about the expression and function of miRNAs in the context of PCNSL.

In PCNSL tumor tissue, the miRNA expression repertoire is different to that found in systemic non-Hodgkin lymphomas (10). miRNAs have also gained increasing interest as biomarkers isolated from tumor tissue, blood or cerebrospinal fluid (CSF) for diagnostic and prognostic purposes (11). A deregulated expression of single miRNAs in the CSF of PCNSL 
patients compared to healthy controls has been observed (12). In contrast, blood-derived miRNA profiles have not yet been assessed in PCNSL patients. Owing to the fact that blood is easily and repeatedly accessible, blood-borne miRNAs are of particular interest as biomarkers.

G-PCNSL-SG1 is the only completed phase III study in PCNSL (13). Using blood samples collected from immunocompetent patients with newly diagnosed PCNSL who entered the trial, we aimed at defining differences in the blood-derived miRNA expression patterns of two cohorts of patients with short- and long-term survival (STS, LTS). All miRNA samples were analyzed using massively parallel, next generation sequencing (NGS) and subsequently validated using quantitative real-time polymerase chain reaction (PCR). We hypothesized that differences in peripheral blood miRNA profiles may represent suitable biomarkers which can be exploited for prognostic purposes and eventually clinical decision making. 


\section{MATERIALS AND METHODS}

\section{Samples}

Blood samples were obtained from patients with confirmed diagnosis of PCNSL at the time of enrolment in the G-PCNSL-SG1 study. Within this trial, all patients initially received HDMTX-based chemotherapy followed by whole brain radiotherapy (WBRT), salvage chemotherapy or observation as defined in the protocol. No maintenance therapy with rituximab or any chemotherapeutic agent was administered (13). Written informed consent was obtained from all patients before blood sampling. Blood was collected using EDTA tubes which were subsequently frozen at $-80{ }^{\circ} \mathrm{C}$ until further processing. In total, 20 patients who succumbed to tumor early in the disease course were chosen for the STS group. Another 20 patients with favourable course were grouped for the cohort of long-term survivors (LTS). A prior power analysis has demonstrated that these cohort sizes at an alpha error probability of 0.05 and a statistical power of 0.95 require an effect size of below 1.2. In previous studies, we usually discovered miRNAs with respective power and these miRNAs were frequently successfully validated by real-time PCR $(14,15)$.

\section{miRNA extraction and NGS}

For the extraction of miRNA, the cellular fractions were pelleted by centrifugation and further processed using the miRNeasy kit (Qiagen GmbH, Hombrechtikon, Switzerland) (16). RNA samples were subjected to analysis by NGS. In brief, $1 \mu \mathrm{g}$ of total RNA per sample was used for library preparation. For quantification, the RNA 6000 Pico Chip on the Bioanalyzer 2100 (Agilent, Santa Clara, CA) was employed. Subsequently, the NEBNext Small Library Prep Kit (New England BioLabs, Beverly, USA) was applied according to the manufacturer's instruction. The resulting libraries were measured with the Bioanalyzer using the DNA 1000 Chip and subsequently pooled in batches of ten samples in equal amounts. Sequencing 
libraries were then clustered with a final concentration of 10 pmol with $1 \%$ PhiX control in one lane each of a $50 \mathrm{bp}$ single read flowcell using the cBot (Illumina). Sequencing of 50 cycles was performed on a HiSeq 2000 (Illumina). De-multiplexing of raw sequencing data and generation of fastq files was done using bcl2fastq 1.8.3.

\section{Statistical analysis}

As a first step, the pre-processing of the Illumina HiSeq raw reads was carried out. In the beginning, the 3 ' adapter sequences were cut using the program fastx_clipper from the FASTX-Toolkit. Subsequently, we applied the miRDeep2 pipeline for retrieving the miRBase counts for release v20 and prediction of novel miRNAs. All further downstream statistical analyses were carried out using the freely available statistical programming language $\mathrm{R}$ (version 3.0.2; http://cran.r-project.org/). Data were normalized by quantile normalization in order to account for systematic shifts in read counts per sample. The resulting data matrix was used as input for all further downstream analyses carried out in R. Prior to employing t-test, the approximate normal distribution of the measured miRNAs was tested by Shapiro-Wilk test followed by adjustment for multiple testing. Next, miRNAs showing a different behaviour between long- and short-time survivors were identified by unpaired two-tailed parametric ttest. Significance values (p-values) obtained for each individual miRNA were adjusted for multiple testing by Benjamini-Hochberg adjustment (17). For novel miRNAs, we additionally calculated significance values using the non-parametric Wilcoxon-Mann-Whitney test, which does not rely on the assumption that data have to be normally distributed. As additional measure, a Receiver Operating Characteristic (ROC) analysis was carried out and the Area Under Curve (AUC) value of miRNAs was computed separately. AUC values are in the range from 0 to 1 , where a value of 0.5 indicates random distribution of variations among STS and LTS, corresponding to the least diagnostic information content of a biomarker. An AUC value 
of 1 means that all expression intensities of the respective miRNA are higher in LTS (upregulated miRNA). Accordingly, an AUC of 0 means that all expression values of the miRNA are higher in STS (down-regulated biomarker).

In addition to this single biomarker analysis, which does not account for potentially improved diagnostic information content by combining the predictive power of different markers, samples were subjected to a classification analysis using Support Vector Machines (SVM) implemented in the R e1071 package (15). For classification, we determined the specificity, sensitivity and accuracy. The specificity corresponds to the percentage of correctly identified controls, i.e., true negatives divided by true negative plus false positives. A true negative (TN) denotes a LTS that is actually recognized, while a false positive (FP) represents a LTS predicted to be a STS. Similarly, the sensitivity corresponds to the percentage of correctly identified STS, i.e., true positives divided by true positives plus false negatives. Here, a true positive (TP) denotes a short-term survivor that is actually recognized, while a false negative (FN) represents a short-term survivor that is wrongly recognized. The accuracy represents the percentage of correct predictions, i.e. $(\mathrm{TN}+\mathrm{TP}) /(\mathrm{TN}+\mathrm{TP}+\mathrm{FN}+\mathrm{FP})$. Following classification, non-parametric permutation tests were carried out in order to check for potential overtraining. Here, the class labels were sampled at random and classifications were carried out using the permuted class labels. All statistical analyses were performed using the R program as previously described (18).

For miRNA enrichment analysis, the tool for annotations of microRNAs (TAM) was applied (19). Specifically, all miRNAs that were influenced by short- or long-term survival were compared to the background set of all miRNAs included in the NGS study. Enrichment was carried out for the precursor molecules and all categories with a p-value $<0.05$ were considered significant if at least 2 miRNAs were located in the respective category. If not indicated otherwise, significance values correspond to raw p-values 


\section{Real-time reverse transcription $(\mathrm{RT})-\mathrm{PCR}$}

An ABI PRISM 7000 Sequence Detection System and TaqMan microRNA assays (Applied Biosystems, Rotkreuz, Switzerland) were used for quantification of miRNA expression by real-time PCR. RNU48 which was established before as an appropriate endogenous control for blood cell-derived miRNAs was used for normalization $(16,20)$. The following conditions were applied for all PCR reactions: 40 cycles of $95^{\circ} \mathrm{C}$ for $15 \mathrm{~s}$ and $60^{\circ} \mathrm{C}$ for $60 \mathrm{~s}$. Data analysis was done using the $\Delta \Delta \mathrm{C}_{\mathrm{T}}$ method for relative quantification as previously specified (21). 


\section{RESULTS}

Prognostic factors that may be helpful to classify patients and adapt treatment decisions are rare in PCNSL. We aimed at defining the potential use of peripheral blood miRNAs as prognostic markers. To this end, we set up two groups of patients who differed significantly in their survival times. The group of patients who died early (STS) had a median OS of 3 months. In contrast, the LTS group had a median OS of 55 months. Importantly, both cohorts were balanced for the well-known prognostic factors age and KPS (5). The median age in the STS group was 64 years, ranging from 53 to 80 years. In the LTS group, median age was 62 (range 42-81). Median KPS at the time of study enrolment was $70 \%$ for both groups. Within the group of short-term survivors, 11/20 patients received only HD-MTX but no further treatment because of rapid tumor progression and subsequent death. From the 20 long-term survivors, 3 received HD-MTX alone, 6 had HD-MTX-based chemotherapy followed by WBRT according to the study protocol and 11 patients were initially treated with HD-MTX and received salvage therapy at tumor recurrence. A detailed overview of patients' characteristics is provided in Suppl. Table 1. In order to report PCNSL biomarker profiles, we first carried out a single marker analysis, followed by machine learning technologies to cluster miRNA profiles. Finally, we confirmed the biomarker candidates by real-time RT-PCR in a validation cohort.

\section{Single marker analysis}

All blood samples from the STS and LTS group were analyzed by NGS. All known miRNAs that are listed in the Sanger miRBase version 20 were analyzed using computational approaches. First, the lowly abundant or not expressed miRNAs (median of 10 raw reads) were excluded from the analysis such that 499 miRNAs remained. A comparison of the 
miRNA expression between the two groups displayed a significant deregulation of 12 miRNAs, reflecting $2.4 \%$ of all 499 expressed miRNAs. In the LTS group, 9 miRNAs were up-regulated while 3 miRNAs were down-regulated. After adjustment for multiple testing, however, none of the miRNAs remained significant. This is likely due to the restricted sample number relative to the very high number of miRNAs. Nevertheless, the raw p-values showed a tendency to accumulate at lower p-values (Fig. 1), potentially indicating significant markers that may be validated by real-time RT-PCR. All significantly deregulated miRNAs identified by NGS are shown together with the respective error bars in Figure 2. As further criterion for the diagnostic performance of the biomarker candidates we evaluated the area under the curve (AUC) in a receiver operator characteristics (ROC) analysis. Especially the three downregulated biomarker candidates showed a high standard deviation leading to limited AUC values, restricting their use as single biomarkers. Within the group of up-regulated miRNAs we found four with particularly high AUC values (> 0.7): miR-151a-5p, miR-151b, miR106a-5p and miR-17-5p. The respective ROC curves are presented in Figure 3A. We also asked whether miRNA expression correlates with therapy response. To this end, we grouped the patients based on their best response to MTX-based therapy in those with complete remission $(n=14)$ and progressive disease $(n=16)$. For this comparison the same calculations as presented above were carried out. Here, we discovered 37 miRNAs with a high AUC value above 0.75 , containing many candidates from the previous analysis including miR-151a-5p, miR-151b, miR-183-3p and miR-96-5p, which are exemplarily shown in Figure 3B. These results indicate that the expression of several miRNAs correlates with response to therapy.

In order to further support our hypothesis that miRNAs are reasonable biomarkers for predicting the survival time of PCNSL patients, we carried out a survival time analysis. To this end, each miRNA was split in a highly expressed and lowly expressed group, where highly expressed means that the respective miRNA for a patient was above the average value. 
For each marker, a survival time analysis was carried out separately. Using the binary variable, we calculated survival curves, which revealed a statistically significant miRNAdependent survival for 15 of the expressed miRNAs (Fig. 4). Since this analysis addresses a similar question as the above described t-tests, the results show a certain overlap such as miR$183-3 p$ that was detected in both approaches.

Further analysis of the NGS dataset demonstrated the presence of short RNA molecules with putative miRNA function that had not been described before. We sorted RNA oligonucleotides, which had to be considered as fragments of mRNAs as outlined in the methods part. The remaining RNA molecules may represent novel miRNA and are identified for the first time within our analysis. The respective marker candidates that deserve further validation were denoted as PCNSL-miR-XXX, where the miRNAs were numbered in increasing order. Many of the putative novel miRNAs were excluded in a filtering step since they mapped to other RNAs. Next, according to the known miRNAs, low abundant miRNA candidates were filtered out such that 49 miRNAs remained. Of the 49 markers, three miRNAs were deregulated between STS and LTS patients including PCNSL-miR-28, PCNSL-miR-901 and PCNSL-miR-904 as determined by t-test, one additional marker, PCNSL-miR-31, was significant according to a non-parametric Wilcoxon-Mann-Whitney test (see Materials and Methods section). Detailed information for these four miRNAs is provided in Suppl. Table 2.

\section{Complex miRNA signatures}

Single deregulated miRNAs from the blood will hardly be useful as prognostic biomarkers. However, the respective markers can be combined in order to reach improved statistical power. To approach complex profiles we first asked whether the miRNAs with diagnostic information content as discovered above may be linked to specific pathological conditions and thereby provide valuable information concerning the underlying pathobiology. Within the 
group of abundantly expressed miRNAs, we observed several molecules that are known to be involved in various physiological and pathological processes. We thus carried out a downstream miRNA enrichment analysis using the tool for annotations of microRNAs (TAM) that includes different miRNA families, data from the human miRNA and disease database (HMDD) (19) and information on various functional classifications of miRNAs. As input for TAM, we used all miRNAs with significance values below 0.1 to increase the sensitivity of our downstream analysis. Altogether, TMA revealed several significantly enriched categories including the subgroup "onco-miRNAs" with highest significance $(\mathrm{p}=0.0007)$, followed by "immune system" and "B cell lymphoma" (p-value of 0.005), as detailed in Suppl. Table 3. The examination of numerous miRNAs allows for the generation of complex miRNA fingerprints in the blood that serve as basis for the classification of the samples as STS or LTS. Using statistical learning techniques, we carried out 25 iterations of a 10-fold crossvalidation and likewise 25 non-parametric permutations with random class labels, i.e. each sample has been assigned to either the short or the long time survivor class. To determine a suitable subset of miRNA biomarkers, we started the training process with the two most significantly deregulated markers and in a stepwise forward approach iteratively increased the number of miRNAs contributing to the classification (in order of increasing p-values according to t-test). This filtering-based feature selection approach, which does not consider correlations between miRNAs, was repeated until all miRNAs were included. Combining multiple miRNAs resulted in an improved classification performance. The respective box plots showing the highly significant results are presented in Figure 5. Here, the three red boxes represent the result of the classification accuracy, specificity and sensitivity. In contrast, the blue boxes can be considered as background value for the classification. Using this procedure, the maximal AUC of the repeated cross-validations was 0.755, being above the values of the most significant single markers as presented above. Although this classification 
performance is not yet ready to allow for clinical application, increased data sets bear the potential to improve the subset selection and classification process substantially.

\section{Real-time PCR validation}

Finally, we aimed at validating the differential expression of known miRNAs as well as putative novel miRNAs between short- and long-term survivors in a second, independent cohort of patients. Again, we set up two groups of patients from the G-PCNSL-SG1 trial differing in the survival time. Median OS in the validation group was 6 months for STS and 35 months for LTS, respectively. We chose 10 deregulated miRNAs identified by NGS for confirmation in the validation cohort including three newly identified putative miRNAs. Using real-time PCR, we observed a consistent deregulation of 8 out of 10 tested miRNAs in the same direction as demonstrated by NGS indicating that similar results can be obtained in a second cohort of patients (Fig. 6). 


\section{DISCUSSION}

Biomarkers that allow for a prognostic classification of patients can be an important tool for optimal patient-adapted therapy and are urgently needed in PCNSL. However, well established prognostic markers in PCNSL are rare. Besides age and performance status, the prognostic score of the International Extranodal Lymphoma Study Group (IELSG) includes elevated serum lactate dehydrogenase (LDH) levels, high CSF protein concentrations, and the involvement of deep regions of the brain (4). However, the most recent prognostic score for PCNSL patients finally only confirmed age and performance status as prognostic factors upon multivariate analysis (5). Consequently, biomarkers from the blood have not been established for PCNSL. Conflicting results were reported for the prognostic suitability of serum LDH levels $(4,22)$, most likely because LDH levels can be influenced by various pathological conditions. In contrast, complex miRNA profiles obtained from the blood are typically disease-specific and can even differ between different pathological conditions from the same organ such as the brain $(16,23)$. Based on these considerations, we examined two groups of patients, which differed in their survival times for our miRNA assessment. Because of the limited number of available blood samples from patients entering the G-PCNSL-SG1 trial, the size of the STS and LTS groups used for the discovery step was restricted to 20 patients each. The STS and LTS groups were balanced for sex and the only repeatedly approved prognostic factors age and performance status (Suppl. Table 1). However, we cannot fully exclude that other variables beyond these parameters may have been different between the two groups. Therefore, the results of our study need to be confirmed in larger patient cohorts, which may help to reduce the risk of other confounding factors.

All analyses were done using NGS, which, in contrast to microarrays or PCR, also allowed detecting novel putative miRNAs, which had not been known before (Suppl. Table 2). Furthermore, NGS enabled the characterization of comprehensive miRNA expression patterns 
consisting of several miRNA, which typically yields higher diagnostic information than a single marker. The NGS approach used for our study revealed several miRNA that are differentially expressed in the blood of STS and LTS patients (Fig. 2). As expected, the differentiation between STS and LTS patients based on single miRNAs (Fig. 3) could be improved when complex miRNA expression patterns were analyzed (Fig. 5) highlighting the advantages of comprehensive miRNA patterns compared to other potential biomarkers which are typically only univariate markers. Survival analyses confirmed the potential of miRNAs as a prognostic tool. In this analysis, where miRNAs were split in low and high abundant, we demonstrated a clear correlation with overall survival as shown for 15 survival curves with a p-value below 0.01 (Fig. 4). Since the STS and LTS groups had substantially different survival times according to patient selection, these findings need to be confirmed in a larger cohort.

Because of the limited number of available blood samples, only a small proportion of patients treated within the G-PCNSL-SG1 trial could be assessed. The rather small size of the training and confirmation group represents a limitation of our analysis. However, 8 out of 10 deregulated miRNA identified by NGS in the training group were confirmed to be differentially regulated in the second set of short- and long-term survivors (Fig. 6). Two miRNAs were non concordant between both technologies and cohorts (upper left quadrant of the figure). Potential reasons for the divergence between real-time PCR and NGS besides the biological diversity include the nucleic acid composition and the secondary structure of miRNAs.

The results of our study stress the suitability of miRNAs as biomarkers in PCNSL. In addition to a previous study, which demonstrated that miRNAs in the CSF can discriminate between samples of PCNSL patients and healthy controls (12), our study reveals that miRNAs may be valuable tools for the prognostic classification of PCNSL patients. Three single miRNAs in the CSF have been assessed for their use as monitoring parameters during the course of the 
disease (24). Compared to CSF-derived molecules, blood-based biomarkers are easier accessible and can be obtained in parallel to routine blood sampling. Whether miRNAs in the blood can be used for the monitoring of PCNSL patients needs to be defined within a larger cohort of patients in a prospective manner. The analysis of miRNA expression patterns can be performed rapidly using NGS, microarrays or PCR. The confirmation of our NGS results by real-time PCR indicates that miRNA-based analyses are feasible with technologies that are broadly available in the clinical routing setting. Overall, the findings of our proof-of-concept study suggest that miRNA expression signatures in the blood are associated with the prognosis of PCNSL patients. Accordingly, such miRNA fingerprints in the blood may allow for a prognostic stratification of PCNSL patients and ultimately help to improve the therapeutic management.

Disclosures: The authors have no disclosures relevant to the manuscript. 


\section{Acknowledgments}

We thank Jasmin Buchs for excellent technical assistance and acknowledge the contributions from all participating sites, patients and caregivers involved in the G-PCNSL-SG1 trial. Furthermore, support by the DKFZ sequencing core facility is gratefully acknowledged. 


\section{REFERENCES}

1. Roth P, Stupp R, Eisele G, Weller M. Treatment of primary CNS lymphoma. Curr Treat Options Neurol 2014;16(1):277.

2. Korfel A, Schlegel U. Diagnosis and treatment of primary CNS lymphoma. Nat Rev Neurol 2013;9(6):317-27.

3. Bessell EM, Graus F, Lopez-Guillermo A, Lewis SA, Villa S, Verger E, et al. Primary non-Hodgkin's lymphoma of the CNS treated with CHOD/BVAM or BVAM chemotherapy before radiotherapy: long-term survival and prognostic factors. Int J Radiat Oncol Biol Phys 2004;59(2):501-8.

4. Ferreri AJ, Blay JY, Reni M, Pasini F, Spina M, Ambrosetti A, et al. Prognostic scoring system for primary CNS lymphomas: the International Extranodal Lymphoma Study Group experience. J Clin Oncol 2003;21(2):266-72.

5. Abrey LE, Ben-Porat L, Panageas KS, Yahalom J, Berkey B, Curran W, et al. Primary central nervous system lymphoma: the Memorial Sloan-Kettering Cancer Center prognostic model. J Clin Oncol 2006;24(36):5711-5.

6. Levy O, Deangelis LM, Filippa DA, Panageas KS, Abrey LE. Bcl-6 predicts improved prognosis in primary central nervous system lymphoma. Cancer 2008;112(1):151-6.

7. Kreher S, Strehlow F, Martus P, Roth P, Hertenstein B, Röth A, et al. Prognostic impact of intraocular involvement in primary CNS lymphoma: experience from the GPCNSL-SG1 trial. Ann Hematol 2014.

8. Shivdasani RA. MicroRNAs: regulators of gene expression and cell differentiation. Blood 2006;108(12):3646-53.

9. Baer C, Claus R, Plass C. Genome-wide epigenetic regulation of miRNAs in cancer. Cancer Res 2013;73(2):473-7. 
10. Fischer L, Hummel M, Korfel A, Lenze D, Joehrens K, Thiel E. Differential microRNA expression in primary CNS and nodal diffuse large B-cell lymphomas. Neuro Oncol 2011;13(10):1090-8.

11. Iorio MV, Croce CM. MicroRNA dysregulation in cancer: diagnostics, monitoring and therapeutics. A comprehensive review. EMBO Mol Med 2012;4(3):143-59.

12. Baraniskin A, Kuhnhenn J, Schlegel U, Chan A, Deckert M, Gold R, et al. Identification of microRNAs in the cerebrospinal fluid as marker for primary diffuse large Bcell lymphoma of the central nervous system. Blood 2011;117(11):3140-6.

13. Thiel E, Korfel A, Martus P, Kanz L, Griesinger F, Rauch M, et al. High-dose methotrexate with or without whole brain radiotherapy for primary CNS lymphoma (GPCNSL-SG-1): a phase 3, randomised, non-inferiority trial. Lancet Oncol 2010;11(11):103647.

14. Keller A, Leidinger P, Bauer A, Elsharawy A, Haas J, Backes C, et al. Toward the blood-borne miRNome of human diseases. Nat Methods 2011;8(10):841-3.

15. Hausler SF, Keller A, Chandran PA, Ziegler K, Zipp K, Heuer S, et al. Whole bloodderived miRNA profiles as potential new tools for ovarian cancer screening. Br J Cancer 2010;103(5):693-700.

16. Roth P, Wischhusen J, Happold C, Chandran PA, Hofer S, Eisele G, et al. A specific miRNA signature in the peripheral blood of glioblastoma patients. J Neurochem 2011;118(3):449-57.

17. Benjamini Y, Drai D, Elmer G, Kafkafi N, Golani I. Controlling the false discovery rate in behavior genetics research. Behav Brain Res 2001;125(1-2):279-84.

18. Keller A, Ludwig N, Comtesse N, Hildebrandt A, Meese E, Lenhof HP. A minimally invasive multiple marker approach allows highly efficient detection of meningioma tumors. BMC Bioinformatics 2006;7:539. 
19. Lu M, Shi B, Wang J, Cao Q, Cui Q. TAM: a method for enrichment and depletion analysis of a microRNA category in a list of microRNAs. BMC Bioinformatics 2010;11:419. 20. Leidinger P, Backes C, Deutscher S, Schmitt K, Mueller SC, Frese K, et al. A blood based 12-miRNA signature of Alzheimer disease patients. Genome Biol 2013;14(7):R78. 21. Roth P, Junker M, Tritschler I, Mittelbronn M, Dombrowski Y, Breit SN, et al. GDF15 contributes to proliferation and immune escape of malignant gliomas. Clin Cancer Res 2010;16(15):3851-9.

22. No_Authors. A predictive model for aggressive non-Hodgkin's lymphoma. The International Non-Hodgkin's Lymphoma Prognostic Factors Project. N Engl J Med 1993;329(14):987-94.

23. Keller A, Leidinger P, Lange J, Borries A, Schroers H, Scheffler M, et al. Multiple sclerosis: microRNA expression profiles accurately differentiate patients with relapsingremitting disease from healthy controls. PLoS One 2009;4(10):e7440.

24. Baraniskin A, Kuhnhenn J, Schlegel U, Schmiegel W, Hahn S, Schroers R. MicroRNAs in cerebrospinal fluid as biomarker for disease course monitoring in primary central nervous system lymphoma. J Neurooncol 2012;109(2):239-44. 


\section{Figure legends}

Fig. 1. Distribution of raw p-values for the deregulation of individual miRNAs. 499 miRNAs were abundant enough to allow for reliable bioinformatics evaluation. Expression levels in the STS $(n=20)$ and LTS $(n=20)$ cohorts were compared by unpaired two-tailed parametric t-test and the number of individual $p$-values falling into the respective segments of width 0.05 was plotted as histogram. The vertical black line denotes the alpha level of 0.05 .

Fig. 2. Median expression of the 12 significantly deregulated markers. Expression levels as determined by NGS are indicated.

Fig. 3. ROC curves for deregulated miRNA candidates. The blue shaded field indicates the 95\% confidence interval for the ROC curve. A. Comparison of STS and LTS patients. B. Analysis of samples from patients achieving a complete remission (CR) compared to patients experiencing progressive disease (PD).

Fig. 4. miRNAs predict survival of PCNSL patients. For each miRNA, a survival time analysis was carried out using the average expression value as cut-off for separation between low and high expression. Survival curves are shown for 15 miRNAs, which allowed a statistically significant separation between short- and long-term survivors. Red color indicates that a miRNAs is highly expressed, while green corresponds to miRNAs with low expression as detailed in the methods section.

Fig. 5. Classification of samples from short- and long-term survivors. Accuracy, specificity and sensitivity were evaluated using repeated runs of 10-fold cross-validation. Red 
boxes correspond to actual classification results while blue boxes correspond to permutation tests used as control.

Fig. 6. Differentially regulated miRNAs identified by NGS can be confirmed in a second cohort of patients. The expression of selected miRNAs that were found to be deregulated by NGS was assessed by qPCR in a second cohort of STS and LTS PCNSL patients. Arrows indicate the shift of regulation in the two cohorts as determined by the two techniques. Novel miRNAs identified with the NGS approach are named with "PCNSL-miR-xxx". 
Figure 1

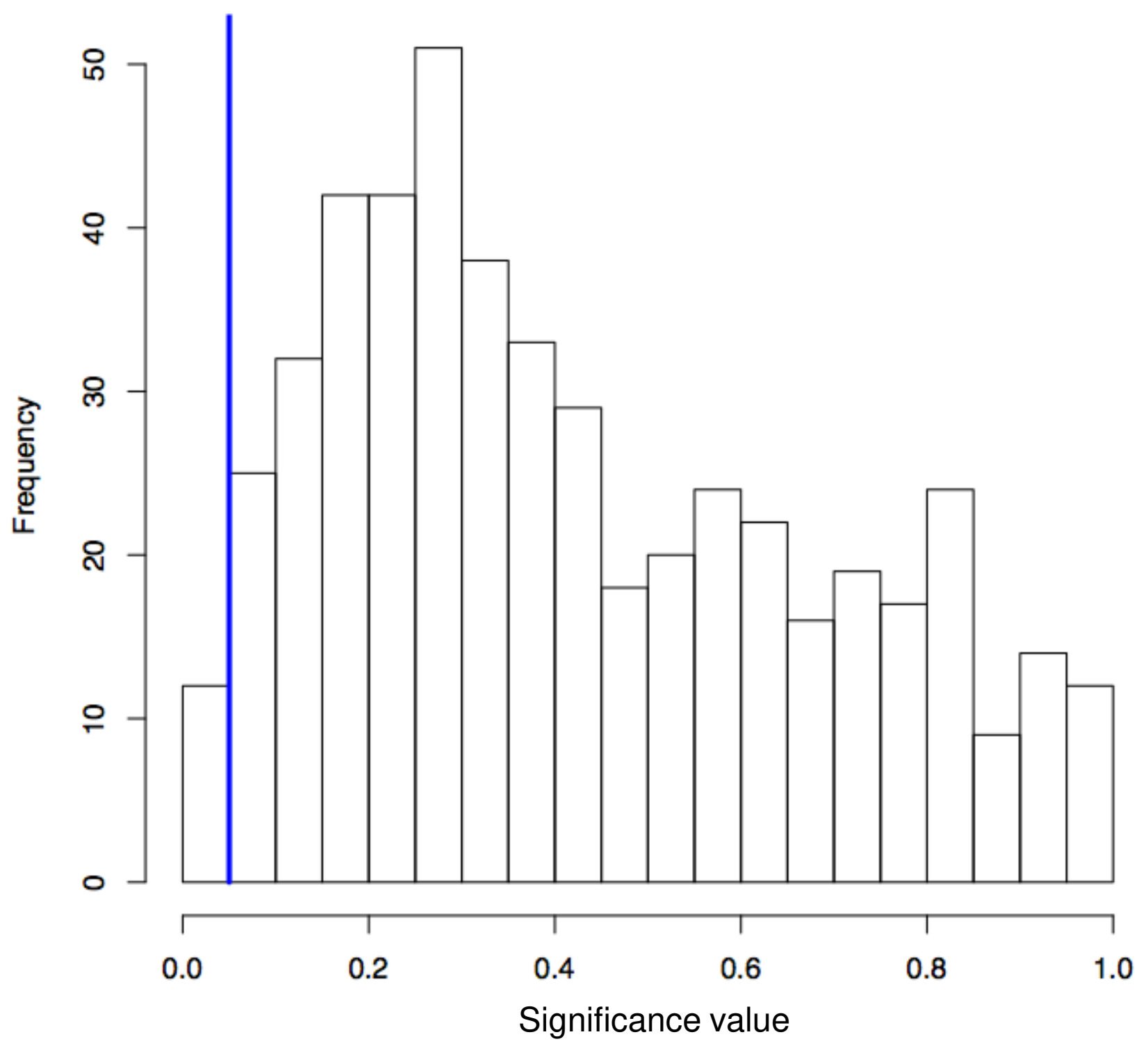


Figure 2

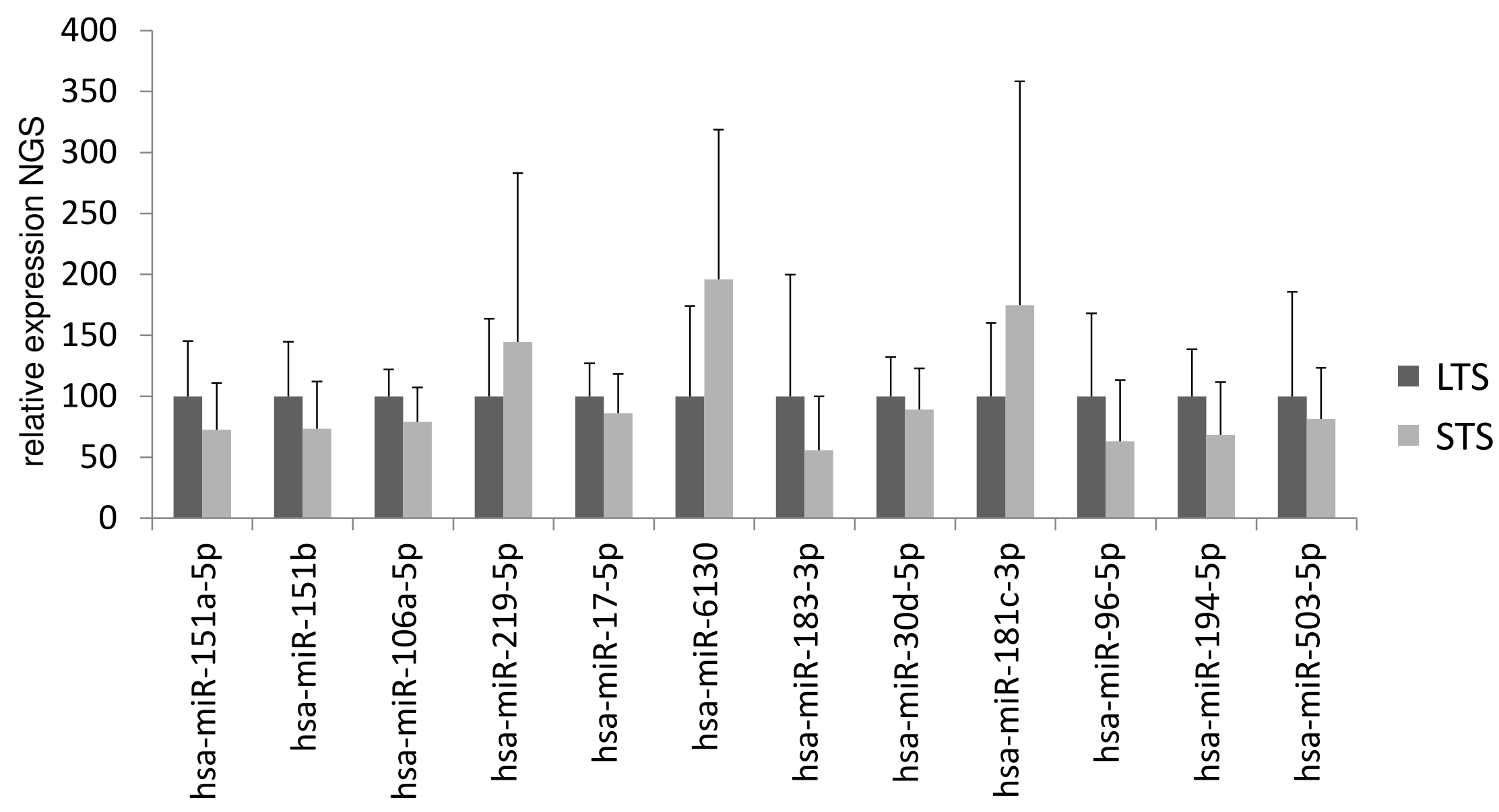




\section{Figure 3}

A
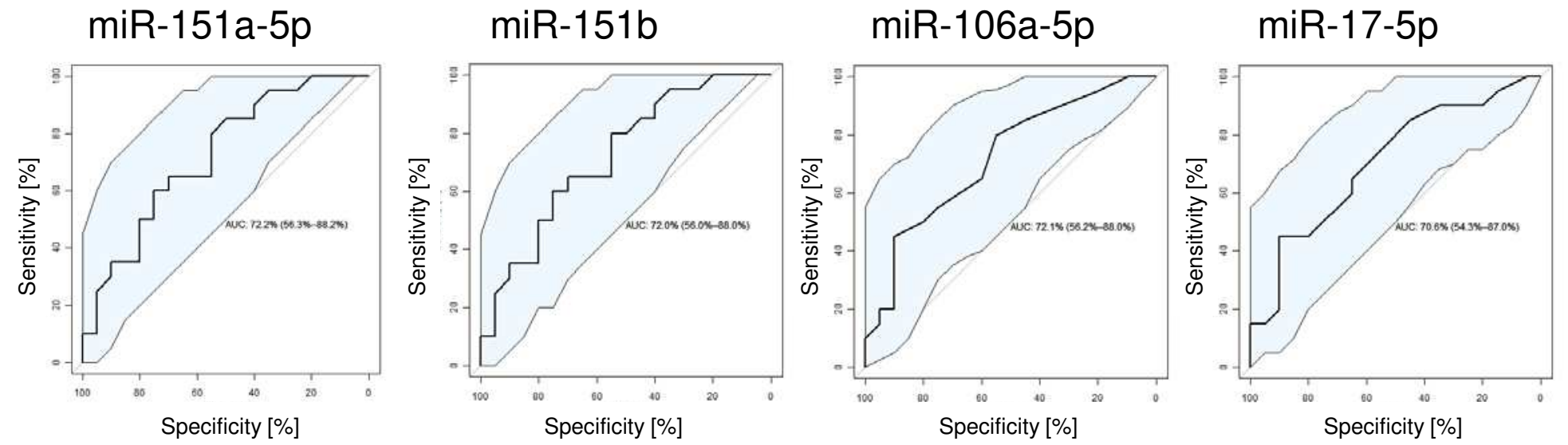

B
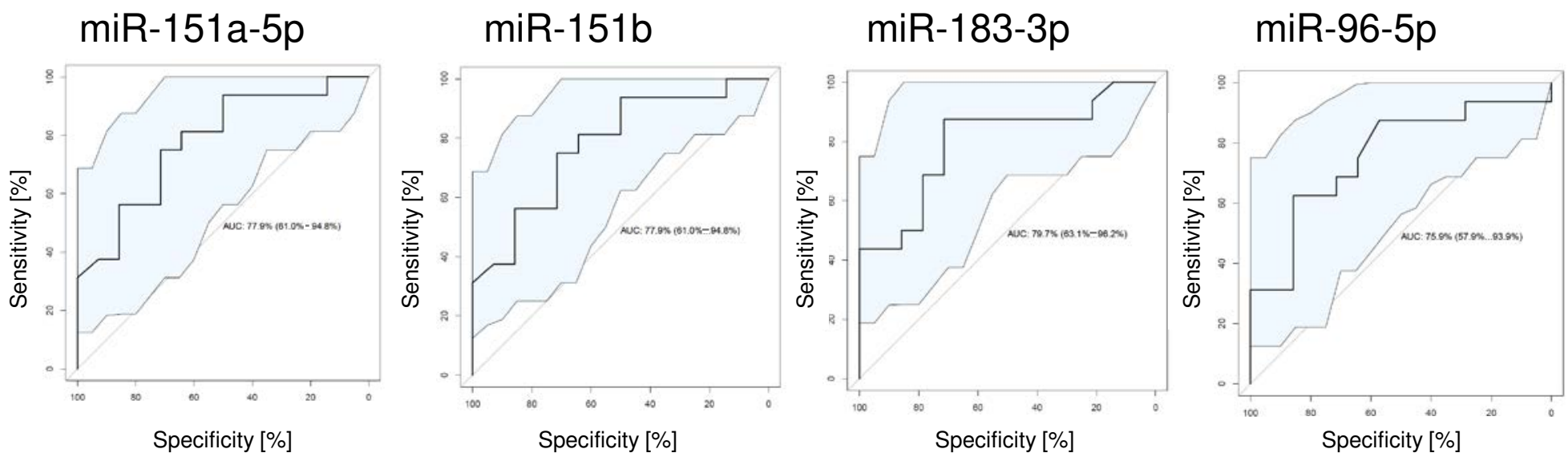


\section{Figure 4}

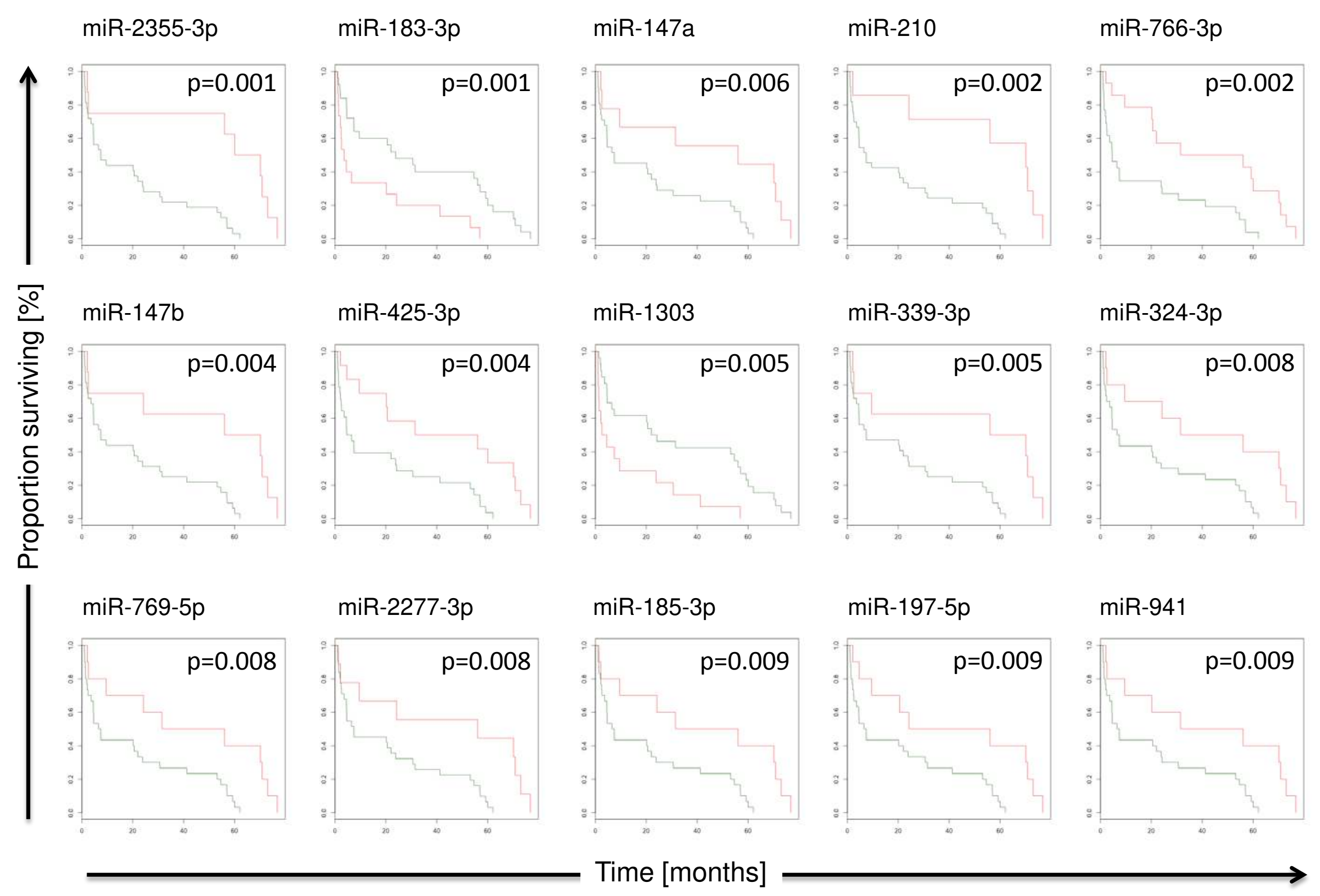


Figure 5

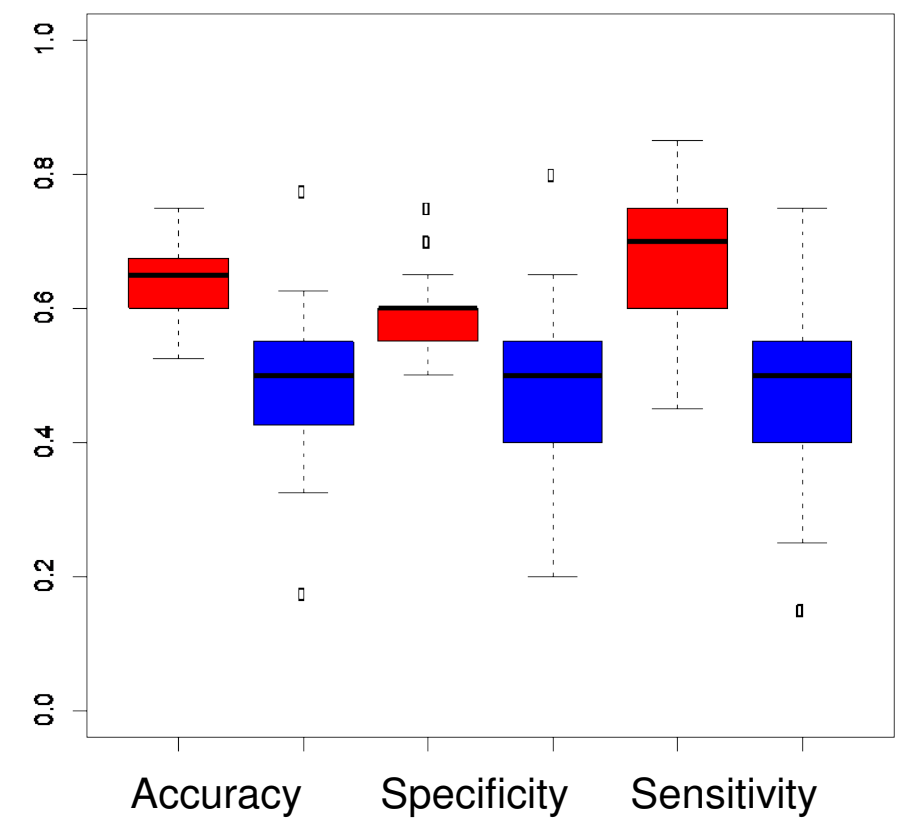


Figure 6

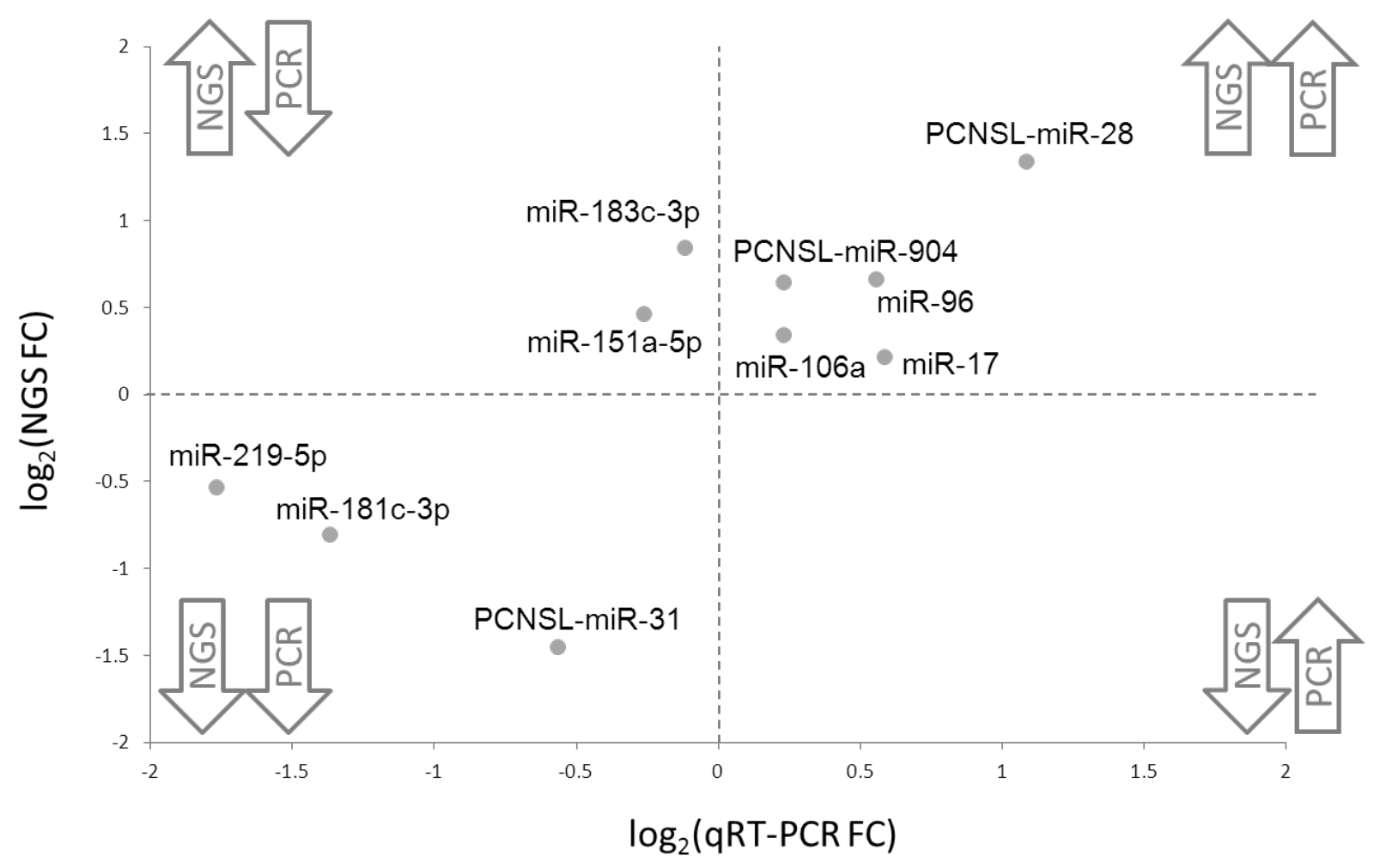

